\title{
Activity and magnetic field structure of the Sun-like planet-hosting star HD 1237
}

\author{
J. D. Alvarado-Gómez ${ }^{1,2}$, G. A. J. Hussain ${ }^{1,4}$, J. Grunhut ${ }^{1}$, R. Fares ${ }^{3}$, J.-F. Donati ${ }^{4,5}$, E. Alecian ${ }^{6,7}$, O. Kochukhov ${ }^{8}$, \\ M. Oksala ${ }^{7}$, J. Morin ${ }^{9}$, S. Redfield ${ }^{10}$, O. Cohen ${ }^{11}$, J. J. Drake ${ }^{11}$, M. Jardine ${ }^{12}$, S. Matt ${ }^{13}$, P. Petit $^{4,5}$, and F. M. Walter ${ }^{14}$
}

1 European Southern Observatory, Karl-Schwarzschild-Str. 2, 85748 Garching bei München, Germany e-mail: jalvarad@eso.org

2 Universitäts-Sternwarte München, Ludwig-Maximilians-Universität, Scheinerstr. 1, 81679 München, Germany

3 INAF-Osservatorio Astrofisico di Catania, via Santa Sofia 87, 95123 Catania, Italy

4 Institut de Recherche en Astrophysique et Planétologie, Université de Toulouse, UPS-OMP, 31400 Toulouse, France

5 CNRS, Institut de Recherche en Astrophysique et Planétologie, 14 avenue Edouard Belin, 31400 Toulouse, France

${ }^{6}$ UJF Grenoble 1 CNRS-INSU, Institut de Planétologie et d'Astrophysique de Grenoble (IPAG) UMR 5274, France

7 LESIA, Observatoire de Paris, CNRS UMR 8109, UPMC, Université Paris Diderot, 5 place Jules Janssen, 92190 Meudon, France

8 Department of Physics and Astronomy, Uppsala University, Box 516, 75120 Uppsala, Sweden

9 LUPM-UMR 5299, CNRS \& Université Montpellier, Place Eugène Bataillon, 34095 Montpellier Cedex 05, France

10 Astronomy Department, Van Vleck Observatory, Wesleyan University, 96 Foss Hill Drive, Middletown, CT 06459, USA

11 Harvard-Smithsonian Center for Astrophysics, 60 Garden Street, Cambridge, MA 02138, USA

12 SUPA, School of Physics and Astronomy, University of St Andrews, St Andrews KY16 9SS, UK

13 Department of Physics and Astronomy, University of Exeter, Stocker Road, Exeter EX4 4SB, UK

14 Department of Physics and Astronomy, Stony Brook University, Stony Brook NY 11794-3800, USA

Received 30 January 2015 / Accepted 13 July 2015

\section{ABSTRACT}

\begin{abstract}
We analyse the magnetic activity characteristics of the planet-hosting Sun-like star, HD 1237, using HARPS spectro-polarimetric time-series data. We find evidence of rotational modulation of the magnetic longitudinal field measurements that is consistent with our ZDI analysis with a period of 7 days. We investigate the effect of customising the LSD mask to the line depths of the observed spectrum and find that it has a minimal effect on the shape of the extracted Stokes $V$ profile but does result in a small increase in the $\mathrm{S} / \mathrm{N}(\sim 7 \%)$. We find that using a Milne-Eddington solution to describe the local line profile provides a better fit to the LSD profiles in this slowly rotating star, which also affects the recovered ZDI field distribution. We also introduce a fit-stopping criterion based on the information content (entropy) of the ZDI map solution set. The recovered magnetic field maps show a strong (+90 G) ring-like azimuthal field distribution and a complex radial field dominating at mid latitudes ( 45 degrees). Similar magnetic field maps are recovered from data acquired five months apart. Future work will investigate how this surface magnetic field distribution affeccts the coronal magnetic field and extended environment around this planet-hosting star.
\end{abstract}

Key words. stars: activity - stars: magnetic field - stars: solar-type - stars: individual: HD 1237

\section{Introduction}

Observational studies of magnetism in late-type stars have evolved dramatically during the past two decades from the classical chromospheric activity diagnostics (e.g. Mount Wilson H-K project, Baliunas et al. 1995) to spectro-polarimetric snapshot surveys (the BCool project, Marsden et al. 2014) and detailed long-term magnetic monitoring (e.g. Morgenthaler et al. 2012). This has been enabled by the advent of improved instrumentation (e.g. ESPaDOnS at CFHT, Donati 2003), together with advanced data analysis techniques for detection (e.g. least squares deconvolution, Donati et al. 1997) and mapping (e.g. Zeeman Doppler imaging, Donati \& Brown 1997) of magnetic fields. These recent studies have opened new possibilities for different areas of astrophysical research, in particular, on dynamo processes and the origin of stellar magnetic fields across the HR diagram (see Donati \& Landstreet 2009). In the case of main sequence solar-type stars, complex and relatively weak $(\ll 1 \mathrm{kG})$ large-scale surface field topologies have been reported. The appearance of ring-like structures of significant (and even dominant) toroidal fields in these stars seems to be connected with the rotation period, hence with the dynamo mechanism behind their generation (Petit et al. 2008).

From the perspective of exoplanet studies, activity-related signatures in late-type stars are known to affect the detection techniques in the form of radial-velocity jitter and photometric flicker (Bastien et al. 2014). By simulating the effects induced by active regions and spots in Sun-like stars, recent tools have been developed to estimate and remove their contribution from the observations (e.g. Dumusque et al. 2014). Other studies have considered the detectability of planets around active cool stars by modelling the stellar activity from recovered starspot and magnetic field maps (Jeffers et al. 2014a; Donati et al. 2014). It is clear that knowing the characteristics of the stellar magnetic field is crucial for addressing the presence of exo-planets in a given system (e.g. the case of $\epsilon$ Eridani, Anglada-Escudé \& Butler 2012; Jeffers et al. 2014b).

In addition, the stellar magnetic field dominates the environment around late-type stars. This includes transient events, such as flares and coronal mass ejections (Shibata \& Magara 2011), and the development of persistent solar-like winds and astrospheres (Wood 2004). These phenomena are known to have a 
profound impact on the structure of exoplanet atmospheres, a critical factor in the habitability of exo-planetary systems (see Vidotto et al. 2013; Cohen et al. 2011b, 2014). They can erode atmospheres through thermal evaporation or non-thermal processes, such as sputtering and ion pick-up. Significant mass loss has been detected for exoplanets that is driven by the stellar wind (Vidal-Madjar et al. 2003; Linsky et al. 2010; Jensen et al. 2012). Models of this interaction require accurate knowledge of the wind properties (Ekenbäck et al. 2010; Sanz-Forcada et al. 2011) and, therefore, of the host-star surface magnetic field. However, given the observational limitations, robust surface field distributions are known for a very limited number of Sun-like planethosting stars (e.g. Fares et al. 2013; Fares 2014). In this context, detailed studies of these systems are very valuable resources not only as direct stellar counterparts of our solar system, but also for the growing interest in finding suitable Earth-like life supporting places in the Universe.

In this article we present our detailed study of one such planet-hosting Sun-like star (HD 1237), in which we investigate the large scale magnetic field and chromospheric activity. This is the first step in characterising the impact of the stellar magnetic field on the circumstellar environment around this system. In particular, the conditions and possible interactions via the magnetically driven stellar wind, with the Jupiter-size exoplanet that comes as close as $0.25 \mathrm{AU}$ in its orbit (Naef et al. 2001). In Sect. 2, we summarise the main properties of the star. Details of the observations and calibration procedures are given in Sect. 3. We present the activity diagnostics and variability in Sect. 4. Section 5 contains a description of the implemented technique for extracting the magnetic field signatures from the spectropolarimetric data. The required steps for the imaging procedure and the resulting surface field maps are presented in Sect. 6. In Sect. 7, we discuss our findings in the context of previous and ongoing studies of solar-type stars. Our main conclusions are summarised in Sect. 8.

\section{HD 1237 stellar properties}

HD 1237 (GJ 3021) is a bright $\left(V_{\text {mag }}=6.58\right)$, Sun-like star (G8V) located about $17.5 \mathrm{pc}$ from the Sun in the southern constellation of Hydrus (Koen et al. 2010). This object is a relatively young $(\sim 0.88 \mathrm{Gyr})$, chromospherically active, and confirmed exoplanet host star. Naef et al. (2001) used the enhanced chromospheric activity to explain the large residuals arising from the best Keplerian orbital solution of the planet $\left(M_{\mathrm{p}} \sin (i)=\right.$ $3.37 \pm 0.14 M_{4}, P_{\text {orb }}=133.7 \pm 0.2$ days, $e=0.51 \pm 0.02$, $a=0.49 \mathrm{AU})$.

Table 1 contains the basic stellar properties of HD 1237 taken from Ghezzi et al. (2010), Torres et al. (2006), and Saffe et al. (2005). Rotation period $\left(P_{\text {rot }}\right)$ estimates are sparse, ranging from $\sim 4.0$ to 12.6 days, as summarised by (Watson et al. 2010). As presented in Sect. 6.1, we obtain $P_{\text {rot }}=7.0 \pm 0.7$ from our observations (Sect. 3 ). In addition, we estimated the radial velocity, $v_{\mathrm{R}}$, and the rotational velocity, $v \sin i$, using an automatic spectral classification tool (MagIcS, Donati et al. 2012) and the fundamental properties of the star (Table 1). Using several of our observed spectra, we found on average a $v \sin i$ of $5.3 \pm 1.0 \mathrm{~km} \mathrm{~s}^{-1}$ and a $v_{\mathrm{R}}$ of $-5.2 \pm 0.2 \mathrm{~km} \mathrm{~s}^{-1}$. Literature values for $v \sin i$ range between $\sim 4.5 \mathrm{~km} \mathrm{~s}^{-1}$ and $5.5 \mathrm{~km} \mathrm{~s}^{-1}$ (Naef et al. 2001; Torres et al. 2006; Schröder et al. 2009). However, as indicated by Naef et al. (2001), the $v \sin i$ value can be over-estimated for metal-rich stars such as HD 1237. For the subsequent analysis, we adopted a $v \sin i$ of $5.3 \mathrm{~km} \mathrm{~s}^{-1}$, which is consistent, within the errors, with the value reported by Naef et al. (2001) and Torres et al. (2006).
Table 1. HD 1237 basic properties.

\begin{tabular}{lcc}
\hline \hline Parameter & Value & Reference \\
\hline S. Type & G8V & Torres et al. (2006) \\
$T_{\text {eff }}[\mathrm{K}]$ & $5572 \pm 40$ & Ghezzi et al. (2010) \\
$\log (g)$ & $4.58 \pm 0.2$ & Ghezzi et al. (2010) \\
$R_{*}\left[R_{\odot}\right]$ & $0.86 \pm 0.07$ & Ghezzi et al. (2010) \\
$M_{*}\left[M_{\odot}\right]$ & $1.0 \pm 0.1$ & Ghezzi et al. (2010) \\
$v \sin i\left[\mathrm{~km} \mathrm{~s}^{-1}\right]^{a}$ & $5.3 \pm 1.0$ & This work \\
$v_{\mathrm{R}}\left[\mathrm{km} \mathrm{s}^{-1}\right]$ & $-5.2 \pm 0.2$ & This work \\
$P_{\text {rot }}[\mathrm{days}]$ & $7.0 \pm 0.7$ & This work \\
$\log \left(L_{\mathrm{X}}\right)$ & $29.02 \pm 0.06$ & Kashyap et al. (2008) \\
Age $[\mathrm{Gyr}]^{b}$ & $\sim 0.88$ & Saffe et al. (2005) \\
\hline
\end{tabular}

Notes. ${ }^{(a)}$ Other reports include $4.5 \mathrm{~km} \mathrm{~s}^{-1}$ (Schröder et al. 2009), $5.1 \pm$ $1.2 \mathrm{~km} \mathrm{~s}^{-1}$ (Torres et al. 2006) and $5.5 \pm 1.0 \mathrm{~km} \mathrm{~s}^{-1}$ (Naef et al. 2001). (b) Age estimates range from 0.15 to $0.88 \mathrm{Gyr}$ using various methods (see Naef et al. 2001; and Saffe et al. 2005). 0.88 Gyr corresponds to the age determined using isochrones.

\section{Observational data}

We obtained observations using the polarimetric mode (Piskunov et al. 2011) of the HARPS echelle spectrograph (Mayor et al. 2003) at the ESO $3.6 \mathrm{~m}$ telescope at La Silla Observatory. The wavelength coverage of the observations range from $378 \mathrm{~nm}$ to $691 \mathrm{~nm}$, with a $8 \mathrm{~nm}$ gap starting at $526 \mathrm{~nm}$.

Data were reduced using the REDUCE package (Piskunov \& Valenti 2002; Makaganiuk et al. 2011), which was modified for the HARPS instrument configuration. This package produces an optimal extraction of the bias-subtracted spectra after flat-fielding corrections and cosmic ray removal have been carried out. The continuum level is determined by masking out the strongest, broadest features (e.g. the Balmer lines) and then fitting a smooth slowly varying function to the envelope of the entire spectrum. Spectra are obtained with resolutions varying from 95000 to 113000 , depending on the wavelength, with a median value of 106000 . Uncertainties are derived for each pixel assuming photon statistics. The star was observed at two epochs separated by 5 months (July and December) in 2012. A summary of the observations is presented in Table 2.

The exposure times listed correspond to one circularly polarised spectrum (Stokes $V$ ), which results from combining four individual sub-exposures using the ratio method. As explained in Donati et al. (1997), the polarisation signal is obtained by dividing spectra with perpendicular (orthogonal) polarisation states (for HARPSpol and Stokes $V: 45^{\circ}, 135^{\circ}, 225^{\circ}$, and $315^{\circ}$, using the quarter waveplate). Additionally, a null-polarisation spectrum is constructed to check for possible spurious polarisation contributions in the observations. More details can be found in Bagnulo et al. (2009). Owing to bad weather conditions, two consecutive Stokes $V$ spectra were added together for the night of 2012 July 23.

\section{Magnetic activity and variability}

To characterise the chromospheric activity level of the star during the observed epochs, we used the CaII H (396.8492 nm) \& $\mathrm{K}(393.3682 \mathrm{~nm})$ lines and the classic Mount Wilson S-index, $S_{\mathrm{MW}}$, defined as

$S_{\text {MW }}=\frac{H+K}{R+V}$.

Here, $H$ and $K$ represent the fluxes measured in each of the $\mathrm{Ca}$ line cores using $0.105 \mathrm{~nm}$ wide spectral windows, and $R$ and $V$ 
Table 2. Journal of observations.

\begin{tabular}{lccccc}
\hline \hline $\begin{array}{l}\text { Date } \\
(2012)\end{array}$ & $\begin{array}{c}\text { HJD } \\
(2400000+)\end{array}$ & UT & $\begin{array}{c}t_{\text {exp }} \\
{[\mathrm{s}]}\end{array}$ & $\begin{array}{c}\text { Stokes } I \\
\text { Peak } S / N\end{array}$ & $\begin{array}{c}\text { Phase } \\
(\Phi)\end{array}$ \\
\hline First epoch & & & & & \\
Jul. 15 & 56123.359 & $08: 04: 36$ & 3600.0 & 955 & 0.000 \\
Jul. 16 & 56124.442 & $10: 03: 43$ & 3600.0 & 1214 & 0.155 \\
Jul. 17 & 56125.399 & $09: 01: 10$ & 3600.0 & 841 & 0.291 \\
Jul. 18 & 56126.361 & $08: 06: 35$ & 3600.0 & 877 & 0.429 \\
Jul. 19 & 56127.440 & $10: 01: 33$ & 3600.0 & 753 & 0.583 \\
Jul. 20 & 56128.356 & $08: 00: 16$ & 3600.0 & 1098 & 0.714 \\
Jul. 21 & 56129.374 & $08: 25: 27$ & 3600.0 & 911 & 0.859 \\
Jul. 22 & 56130.438 & $09: 47: 58$ & 4800.0 & 1092 & 1.011 \\
Jul. 23 & 56131.397 & $08: 32: 33$ & 6680.0 & 660 & 1.148 \\
Jul. 31 & 56139.314 & $06: 49: 52$ & 4800.0 & 926 & 2.280 \\
Aug. 02 & 56141.303 & $06: 32: 21$ & 5000.0 & 1040 & 2.564 \\
Second epoch $(20.29+$ rotation cycles since Jul. 15 2012) & \\
Dec. 04 & 56265.045 & $00: 35: 27$ & 2800.0 & 1205 & 0.000 \\
Dec. 05 & 56266.045 & $00: 35: 21$ & 2800.0 & 964 & 0.142 \\
Dec. 06 & 56267.044 & $00: 34: 40$ & 2800.0 & 1018 & 0.285 \\
Dec. 07 & 56268.044 & $00: 33: 59$ & 2800.0 & 722 & 0.428 \\
\hline
\end{tabular}

Notes. The columns contain the date, the corresponding Heliocentric Julian Date (HJD), the start time of the observations in UT, the exposure times, and the Stokes $I$ peak signal-to-noise ratio $(\mathrm{S} / \mathrm{N})$. The rotational phase $(\Phi)$ listed in the last column is calculated using the rotation period derived in this work $\left(P_{\text {rot }}=7.0 \mathrm{~d}\right) .{ }^{(a)}$ The listed values correspond to two spectro-polarimetric exposures merged in a single observation due to bad weather conditions.

are the fluxes measured in the continuum over $2 \mathrm{~nm}$ windows centred at $390.1 \mathrm{~nm}$ and $400.1 \mathrm{~nm}$, respectively, on both sides of the CaII region.

\subsection{Index calibration}

To compare the activity level of HD 1237 with other stars, we need to convert the measured HARPS S-index, $S_{\mathrm{H}}$, to the Mount Wilson scale. For this, we require a calibration factor, $\alpha$, which is an instrument-dependent quantity that linearly relates the values for the classic $S_{\mathrm{MW}}$ and the HARPS fluxes $H, K, R, V$ :

$S_{\mathrm{MW}}=\underbrace{\alpha\left(\frac{H+K}{R+V}\right)_{\mathrm{H}}}_{S_{\mathrm{H}}}$.

We estimated $\alpha$ by including a set of stars with previous measurements of chromospheric activity via $S_{\mathrm{MW}}$ (Santos et al. 2000) and within the HARPS observations' database. The spectral type of the reference stars and their reported $S_{\mathrm{MW}}$ values are listed in Table 3 . The linear relation between $S_{\text {Mw }}$ and the HARPS fluxes is plotted in Fig. 1. The derived calibration factor, within the $1 \sigma$ uncertainty, is

$\alpha=15.39 \pm 0.65$.

All spectra were co-aligned using a high-S/N HARPS solar spectrum as reference. We estimate a 5\% typical error size in our HARPS flux measurements based on the possible differences in the continuum normalisation, which was performed in the same way for all the stars in the calibration. Therefore, the errors in $S_{\mathrm{H}}$ are dominated by the conversion procedure.

We proceed with the estimation of the activity index $S_{\mathrm{H}}$, with the corresponding indicators $R_{\mathrm{HK}}$ (Middelkoop 1982) and $R_{\mathrm{HK}}^{\prime}$ (Noyes et al. 1984), which account for colour and photospheric correction, respectively.
Table 3. Stars included in the $\alpha$ calibration.

\begin{tabular}{llcl}
\hline \hline Name & S. type & $S_{\mathrm{MW}}$ & $\sigma_{\mathrm{MW}}$ \\
\hline HD 1835 & G3V & 0.364 & 0.024 \\
HD 10700 & G8.5V & 0.173 & 0.004 \\
HD 22049 & K2Vk & 0.515 & 0.026 \\
HD 23249 & K1III-IV & 0.150 & 0.012 \\
HD 26965 & G9III-IV & 0.208 & 0.018 \\
HD 30495 & G1.5V & 0.292 & 0.016 \\
HD 61421 & F5IV-V & 0.187 & 0.010 \\
HD 76151 & G3V & 0.262 & 0.018 \\
HD 115617 & G7V & 0.161 & 0.003 \\
HD 149661 & K2V & 0.356 & 0.042 \\
HD 152391 & G8.5Vk & 0.392 & 0.030 \\
HD 155885 & K1V & 0.400 & 0.020 \\
\hline
\end{tabular}

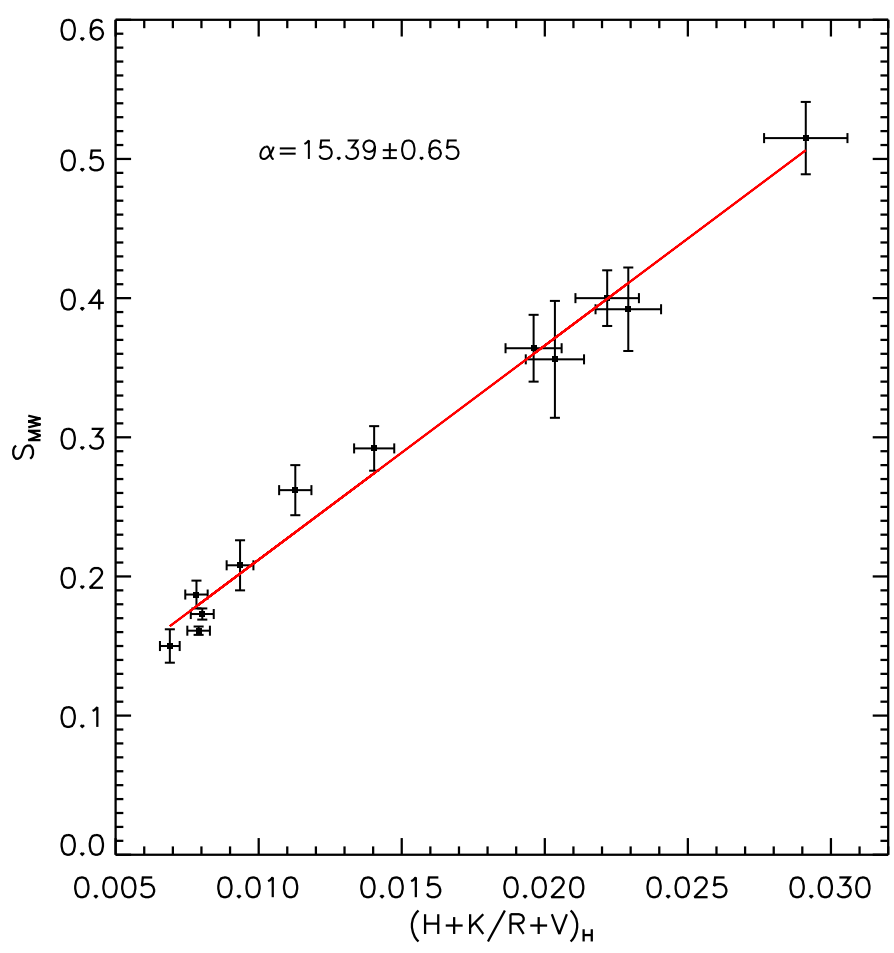

Fig. 1. Linear fit between Santos et al. (2000) $S_{\mathrm{MW}}$ values and the HARPS fluxes $\left(\frac{H+K}{R+V}\right)_{\mathrm{H}}$. A $5 \%$ error is estimated in our HARPS flux measurements.

\subsection{Activity indicators}

Figure 2 shows the cores of the CaII $\mathrm{H}$ and $\mathrm{K}$ lines in the HARPS normalised spectra of HD 1237 for different observations, which are compared with the mean profile (red) derived from the entire dataset. For the averaging procedure we take the slight differences in the wavelength range into account from each observation by an interpolation procedure to match the largest wavelength data points in the observed spectra. A high-S/N HARPS solar spectrum ${ }^{1}$ is shown as reference.

The upper plot shows one observation at a later epoch (2012 Dec 7), where it is possible to observe a variation in the line profile. This is interpreted as a slight change in the chromospheric and photospheric activity of the star in comparison with the mean behaviour of the red line (which is dominated by profiles from the first epoch), especially in the $\mathrm{H}$ line (Fig. 2, right panel). The K-line region of the spectrum contained more noise.

1 S/N: 347 at $550 \mathrm{~nm}$, date: 2007 Apr 12 - low activity period. 

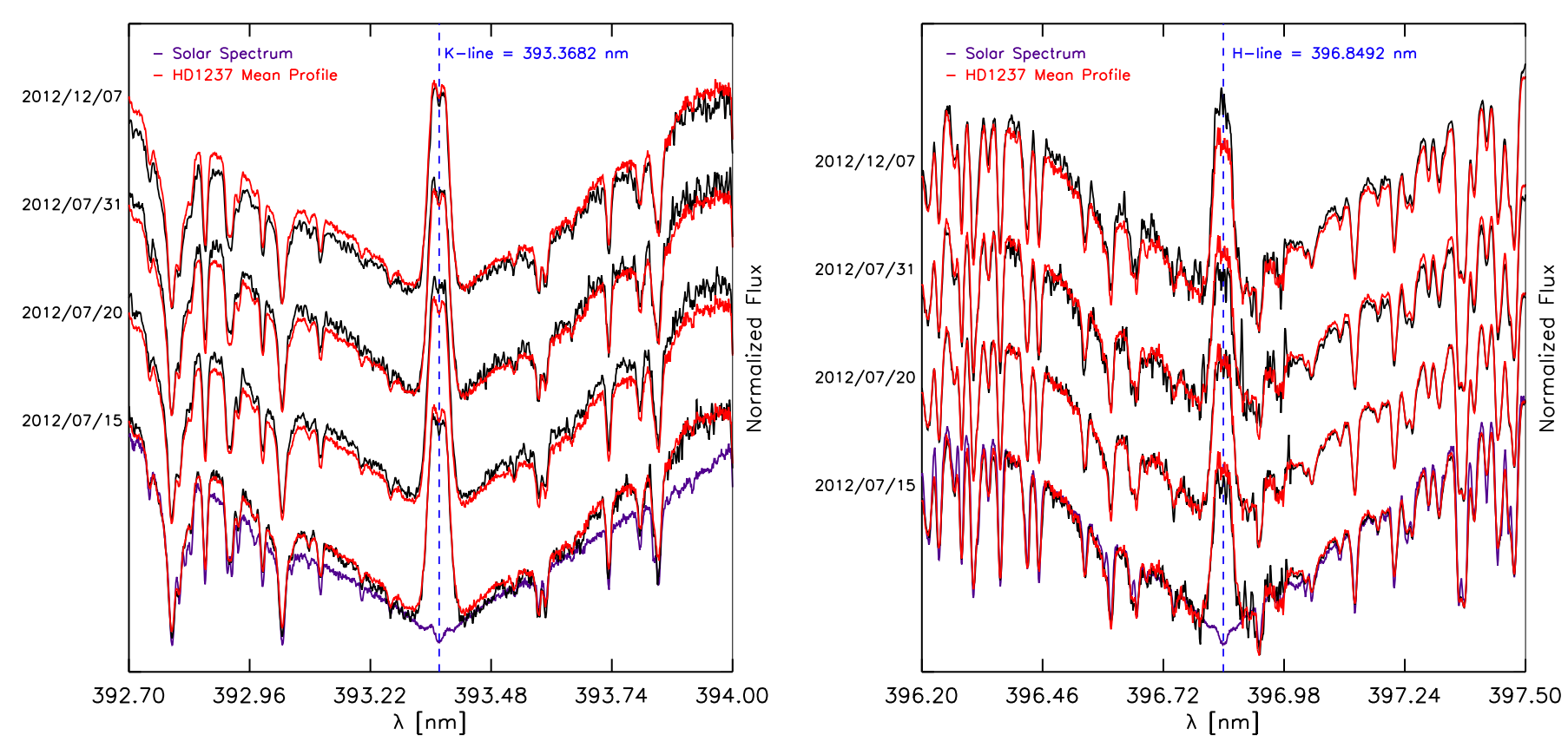

Fig. 2. Core regions of the CaII K (left) and H (right) lines of HD 1237. Four spectra from our sample are plotted with the corresponding dates on the left $y$-axis and vertically shifted ( 0.25 units) for visualisation purposes. The black spectrum at the top was taken at a much later epoch (2012 Dec. 07). The red line shows the mean profile for the entire dataset, while the purple line is a HARPS solar spectrum used as reference.

No change in the activity level of the star is visible in this particular line.

Figure 3 shows the measured HARPS fluxes, $(H+K) /(R+V)$, for each observation starting from 2012 Jul 15 (vertical blue line, HJD $=2456123.5$ ). The $x$-axis units correspond to days after this initial date. The activity of the star showed a marginal variation, in a similar way as in the first epoch. This may be due to the rotation of active regions over the stellar surface. The similarities between the activity levels between both epochs could be an indication of a stable large-scale magnetic field configuration.

The chromospheric activity level of the star can be quantified by using Eq. (2) and the derived value for $\alpha$ in Eq. (3). We estimate an average value of $S_{\mathrm{H}} \simeq 0.46 \pm 0.02$ for the available observations of HD 1237. Similar activity levels have been reported for the Sun-like star $\xi$ boo A $\left(T_{\text {eff }}=5600 \mathrm{~K}, P_{\text {rot }}=6.4\right.$ days, Age: 0.2 Gyr, Mamajek \& Hillenbrand 2008; Morgenthaler et al. 2012). As a reference value, the Solar S-index is $S_{\odot} \simeq$ 0.1783 with a variation of $\sim 0.02$ from solar maximum to minimum (Lockwood et al. 2007).

Using the mean derived values of the S-index, we can now apply a transformation to obtain the parameter $R_{\mathrm{HK}}$, which takes the colour of the star in the activity estimation into account (Middelkoop 1982), Here $R_{\mathrm{HK}}$ is defined as

$R_{\mathrm{HK}}=\left(C_{\mathrm{CF}}\right)\left(S_{\mathrm{H}}\right)\left(1.34 \times 10^{-4}\right)$,

where $C_{\mathrm{CF}}$ is a colour-dependent function. For main sequence stars with $0.3 \leq(B-V) \leq 1.6, C_{\mathrm{CF}}$ is given by

$\log \left(C_{\mathrm{CF}}\right)=0.25(B-V)^{3}-1.33(B-V)^{2}+0.43(B-V)+0.24$.

Noyes et al. (1984) derived an expression in order to include photospheric corrections to the CaII core fluxes, $R_{\mathrm{HK}}^{\prime}$, which is written as

$R_{\mathrm{HK}}^{\prime}=R_{\mathrm{HK}}-R_{\mathrm{phot}}$,
Table 4. Average activity indicators for HD 1237.

\begin{tabular}{ccc}
\hline \hline$\left\langle S_{\mathrm{H}}\right\rangle$ & $\log \left(R_{\mathrm{HK}}\right)$ & $\log \left(R_{\mathrm{HK}}^{\prime}\right)$ \\
\hline $0.462 \pm 0.019$ & $-4.29 \pm 0.04$ & $-4.38 \pm 0.05$ \\
\hline
\end{tabular}

with $R_{\text {phot }}$ expressed also as a function of $(B-V)$ :

$\log \left(R_{\text {phot }}\right)=-4.898+1917(B-V)^{2}-2.893(B-V)^{3}$,

and valid in the range of $0.44<(B-V) \lesssim 1.0$. Table 4 summarises the activity indicators for the available observations of HD 1237. The errors quoted are the mean measurement errors for these quantities. The $B$ and $V$ magnitudes were taken from the HiPPARCOS catalogue $(B=7.335 \mathrm{mag}, V=6.578 \mathrm{mag}$, Koen et al. 2010). High activity levels are related to magnetic fields on the stellar surface. These magnetic signatures are encoded in the polarised spectra of the star and is discussed in the next section.

\section{Magnetic field signatures}

The signal-to-noise ratio $(\mathrm{S} / \mathrm{N})$ in the observations is not high enough to detect magnetically induced spectro-polarimetric signatures in single lines. However, by applying a multi-line technique, e.g. least squares deconvolution (LSD, Donati et al. 1997 ), it is possible to increase the $\mathrm{S} / \mathrm{N}$ by a factor of $\sim 50-100$, adding-up the signal from thousands of spectral lines over the entire spectral range (for HARPS: 378-691 nm), enhancing our sensitivity to magnetic signatures in the observations (see Kochukhov et al. 2010 for a recent review of the LSD technique). This procedure requires a photospheric model (line list) matching the spectral type of our target star. This is done using an atomic line list database ${ }^{2}$ (Kupka et al. 2000) and the

\footnotetext{
2 http://vald.astro.uu.se/ - Vienna Atomic Line Database (VALD3).
} 

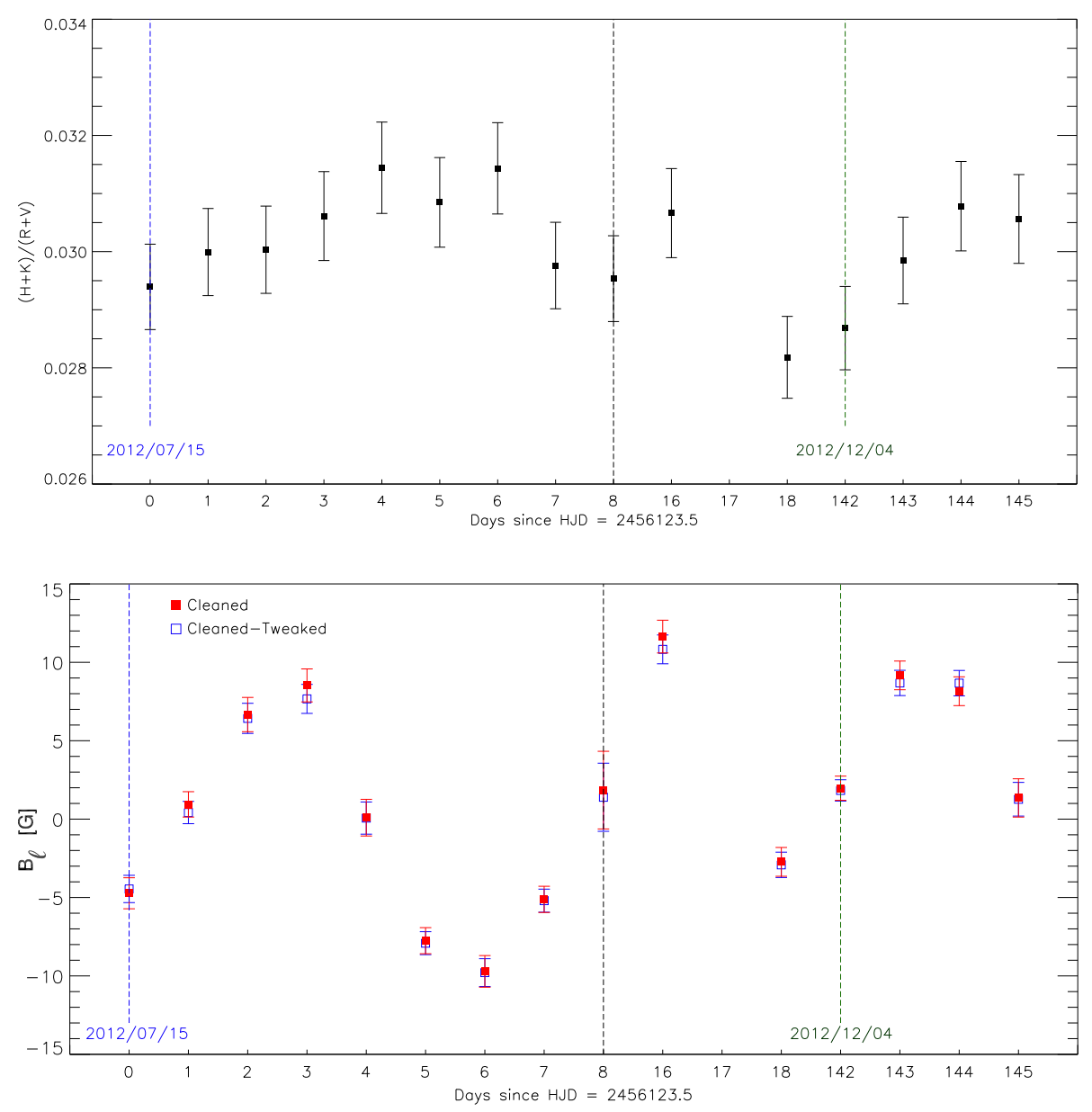

Fig. 3. HARPS fluxes, $(H+K) /(R+V)$, for the available observations of HD 1237. The blue and green vertical lines denote the beginning of each observed epoch. The $x$-axis contains the number of days since the Heliocentric Julian Date (HJD $=2456123.5$ ) of the first observation (2012 Jul. 15). The last two data points of the first epoch are unevenly distributed (after the vertical black line). We estimate a $5 \%$ error in our measurements.
Fig. 4. Calculated $B_{\ell}$ for the available observations of HD 1237. The blue and green vertical lines denote the beginning of each observed epoch. The $x$-axis contains the number of days since the Heliocentric Julian Date (HJD $=2456123.5$ ) of the first observation (2012 Jul. 15). The last two data points of the first epoch are unevenly distributed (after the black line). Colours indicate the line mask used for the LSD procedure. stellar fundamental properties listed in Table 1. We assumed a micro-turbulence parameter of $1.3 \mathrm{~km} \mathrm{~s}^{-1}$ (Ghezzi et al. 2010) and solar abundance for the photospheric line list that included $\sim 15000$ lines within the HARPS spectral range.

From this initial photospheric line list, we generated two different masks used with the LSD calculation. In the first mask the strong lines (and lines blended with these lines) that form in the chromosphere or that break the basic assumptions of LSD (e.g. $\mathrm{Ca}$ II $\mathrm{H} \& \mathrm{~K}, \mathrm{H} \alpha$ ) are removed (cleaned mask). This reduced the number of lines included to $\sim 11000$. After the mask cleaning, a numerical routine based on the Levenberg-Marquardt, non-linear least-squares algorithm from the MPFIT library (Moré 1978; Markwardt 2009) is applied to fit the line mask to the observed Stokes $I$ spectrum after adjusting the individual depths of the spectral lines (cleaned-tweaked mask). This is performed through the entire HARPS wavelength coverage. This step is more commonly carried out when applying LSD to hot (OBtype) stars that have fewer lines (e.g. Neiner et al. 2012). Finally, LSD was applied to the spectro-polarimetric data using the final masks, generating in this way a single, averaged line profile per observation (LSD Stokes $I, V$, and diagnostic N profiles). A velocity step $\Delta v=1.4 \mathrm{~km} \mathrm{~s}^{-1}$ was used to construct the LSD profiles. This velocity spacing considers two pixels per spectral element of the instrument (in the case of HARPSpol, $R=2.5 \mathrm{~km} \mathrm{~s}^{-1}$ and 3.4 px per resolution element).

Both procedures lead us to consistent results in the obtained LSD signatures of the star. While no clear change is observed in the null polarisation check, subtle qualitative differences appear in both Stokes profiles, in the sense that the cleaned-tweaked mask seems to get a broader unpolarised profile with a slightly weaker signature in the circular polarised profile, in comparison with the clean line mask. These minimal shape differences in the LSD line profiles can have a much greater effect in hot stars where fewer lines are generally available. On the other hand, the resulting $\mathrm{S} / \mathrm{N}$ of the LSD profiles for each individual observation was systematically higher using the cleaned-tweaked mask than in the cleaned case, despite the same number of spectral lines in their masks (11048). On average, a $7 \%$ increase was obtained in the $\mathrm{S} / \mathrm{N}$ of the LSD profiles with the cleaned-tweaked mask.

\subsection{Longitudinal magnetic field}

Using the derived Stokes $I$ and $V$ LSD profiles, it is possible to obtain information about the surface averaged longitudinal magnetic field $\left(B_{\ell}\right)$. Following Donati \& Landstreet (2009), an estimate of $B_{\ell}$ (in $\mathrm{G}$ ) is given by

$$
B_{\ell}=-714 \frac{\int v \mathrm{~V}(v) \mathrm{d} v}{\lambda \bar{g} \int[1-\mathrm{I}(v)] \mathrm{d} v},
$$

where the radial velocity shift $v$ (in $\mathrm{km} \mathrm{s}^{-1}$ ) is measured with respect to the average line derived from LSD with central wavelength $\lambda$ (in $\mu \mathrm{m}$ ) and mean Landé factor $\bar{g}$. As this measurement is an integrated quantity over the visible surface, it cannot provide complete information for stars that host complex largescale magnetic fields. From multiple measurements of $B_{\ell}$ taken over a stellar rotation period, it is possible to gain a first insight into inhomogeneities of the disk-integrated magnetic field. It is thus also possible to estimate the stellar rotation period, using 

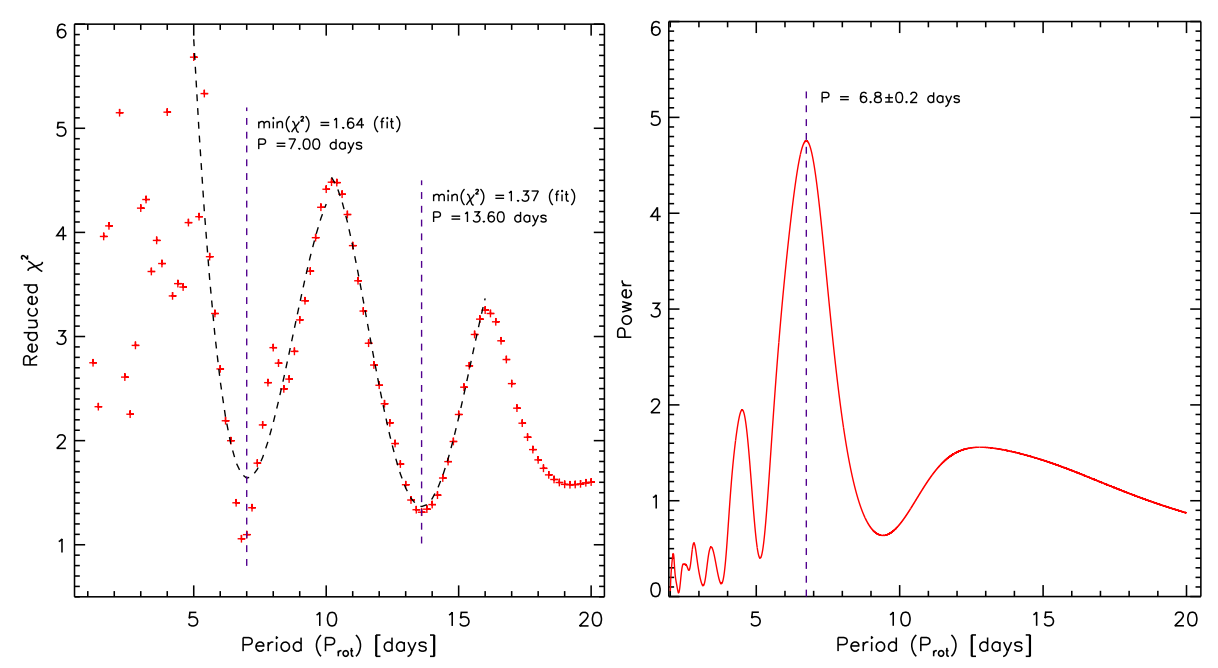

Fig. 5. Left: minimisation results for HD 1237 showing the reduced $\chi^{2}$ as a function of $P_{\text {rot }}$. The black segmented lines corresponds to a fourth-order polynomial fit. Right: power spectrum obtained from the Lomb-Scargle periodogram analysis on the temporal variation of $B_{\ell}$ (Fig. 4). the modulation in a time series of $B_{\ell}$ measurements, provided that they span more than one rotation period.

Figure 4 shows the measurements of $B_{\ell}$ for the available observations of HD 1237. The integration was centred on the radial velocity of the star $\left(-5.2 \mathrm{~km} \mathrm{~s}^{-1}\right)$ and covered the entire Stokes $V$ signature $\left( \pm 12.5 \mathrm{~km} \mathrm{~s}^{-1}\right)$. The uncertainties were computed from standard error propagation from the spectra. The colours denote the line mask used in the LSD procedure, showing that the slight differences in each of the measurements are consistent within the errors. This implies that either set of LSD profiles can be used to obtain robust longitudinal magnetic field measurements. As the two sets of profiles are so similar, there is only a difference in $\mathrm{S} / \mathrm{N}$ without any noticeable impact on the maps structure.

Reflecting the additive nature of the chromospheric emission, $B_{\ell}$ shows a clearer rotational variation in comparison with $S_{\mathrm{H}}$ (Fig. 3), in contrast to the mixed polarity effects of the magnetic field. The behaviour of the longitudinal field is consistent throughout the observed epochs with an varying amplitude of roughly $\sim 10 \mathrm{G}$. This value is somewhat higher than the solar value $\left(B_{\ell}<4\right.$ G, Daou et al. 2006; Kotov et al. 1998) and than the average value from snapshot observations of other Sunlike stars of the same and different spectral types (3.3, 3.2, and 5.7 G for F, G and K-dwarfs respectively, Marsden et al. 2014). However, caution is advised in these averaged comparisons, given the rotational variability of $B_{\ell}$ and the nature of a snapshot survey. Similar variations have been observed in the long-term monitoring of the active Sun-like star $\xi$ Boo A (Morgenthaler et al. 2012).

\section{Surface magnetic field mapping}

\subsection{Optimal line profile and stellar parameters}

We reconstructed surface magnetic field maps by applying the tomographic inversion technique of Zeeman Doppler imaging (ZDI; Vogt et al. 1987; Semel 1989). As described by Hussain et al. (2000), ZDI has been used to recover magnetic field maps on the surfaces of stars ranging from $\mathrm{T}$ Tauri stars to binary systems (e.g. Barnes et al. 2004; Dunstone et al. 2008). The code recovers the magnetic flux distribution across the stellar disk, modulated by the stellar rotation, by using time series of photospheric absorption line profiles (LSD Stokes $I$ ) and circularly polarised profiles (LSD Stokes $V$ ).

To recover reliable magnetic field maps, it is necessary to properly model the LSD Stokes $I$ and $V$ profiles and their temporal variation. To do this, good constraints should be obtained on the local line profile description and the stellar parameters. Two different synthetic line shapes were tested: a Gaussian profile, which is commonly used in magnetic field studies in Sun-like stars (e.g. Boro Saikia et al. 2015), and a Milne-Eddington profile, fitted to a solar LSD profile derived from a high-S/N HARPS spectrum. This last approach was previously considered in ZDI of accreting $\mathrm{T}$ Tauri stars (Donati et al. 2008a) and M dwarfs (Morin et al. 2008). For both cases, we assumed a linear dependence of the continuum limb darkening with the cosine of the limb angle (slope $u \simeq 0.65$, Sing 2010).

In addition, we estimated the rotational period $P_{\text {rot }}$ differential rotation profile (e.g. $\Omega(l)=\Omega_{\mathrm{eq}}-\mathrm{d} \Omega \sin ^{2}(l)$, see Petit et al. 2002) and inclination angle $i$ of the star. This is done by generating a grid of ZDI models that covers a range of values for the involved quantities and minimising the reduced $\chi^{2}$ from synthetic line profile fitting (see Collier Cameron 1995; Hussain et al. 2009). Figure 5 (left) shows the results of the minimisation analysis over $P_{\text {rot }}$. As mentioned in Sect. 2, the rotation period of HD 1237 is not well known. Our analysis shows the harmonic behaviour of this parameter with a fundamental value of $\sim 7.0$ days. We estimate a $10 \%$ error for this period determination, given the width of the minima in the left-hand panel of Fig. 4. This estimate is consistent with the high activity level measured in this star (Sect. 4). For completeness, we performed a Lomb-Scargle periodogram, using the first epoch dataset, on the temporal variations of $B_{\ell}$ (Fig. 4). Figure 5 (right) shows the obtained power spectrum, having a best-fit period of $P_{\text {rot }}=6.8 \pm 0.2$ days with an associated $P$-value statistic of $5.18 \times 10^{-5}$ (Zechmeister \& Kürster 2009).

No good constraints were obtained by $\chi^{2}$ minimisations for the differential rotation parameters and the inclination angle of the star. Therefore, no differential rotation profile was included in the mapping procedure. For the inclination angle, we considered the expected value from rigid body rotation, i.e. $\sin i=\left(P_{\text {rot }} \cdot v \sin i\right) /\left(2 \pi R_{*}\right)$. Given the uncertainties of the involved quantities (see Table 1), the inclination angle should lie somewhere between $\sim 40-60^{\circ}$. We performed reconstructions for 40,50 , and $60^{\circ}$ and find no substantial differences between these magnetic field reconstructions. We present here the maps obtained assuming a $50^{\circ}$ inclination angle.

Finally, we compared the optimum stellar and line parameters associated with the LSD profiles produced using both masks (cleaned mask and clean-tweaked mask). No significant 


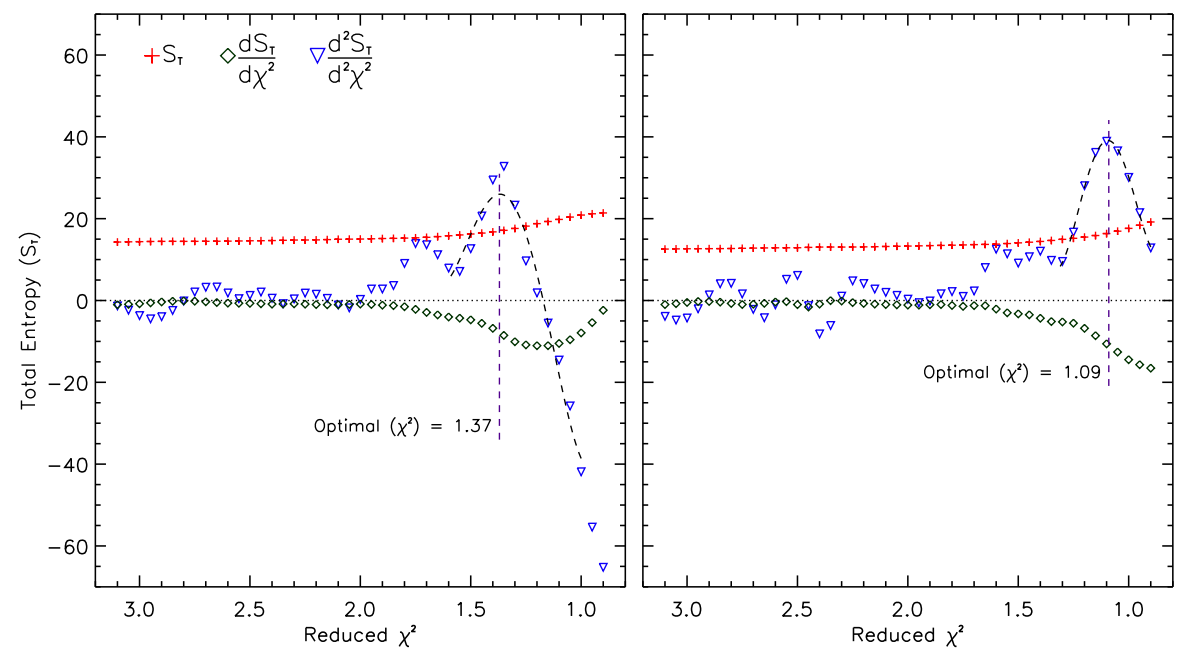

Fig. 6. Optimal $\chi^{2}$ selection criteria applied to the ZDI solution set of HD 1237. Each panel contains the results for the July dataset using the Gaussian (left) and Milne-Eddington (right) line profiles. The red symbols show the behaviour of the total entropy content $S_{\mathrm{T}}$ as a function of the reduced $\chi^{2}$. Each point corresponds to a converged ZDI solution. Green and blue symbols represent the first and second derivatives as indicated. A fourth-order polynomial fit has been applied (segmented line) to find the value for which the rate of change in the information growth (second derivative) in the ZDI solution set is maximised. The optimal fit level is indicated in each case. difference is found in the optimal parameters. In the subsequent analysis, therefore, we only consider the LSD profiles produced using the cleaned-tweaked mask.

\subsection{Optimal fit quality: entropy content in ZDI maps}

The last of part of the analysis corresponds to the selection of the optimal fit quality (reduced $\chi^{2}$ ), of the model with respect to the observations. In principle, the recovered field distribution and associated profiles should try to achieve the lowest possible value of $\chi^{2}$. Still, given the limitations of the observations and the ZDI technique (S/N, spatial resolution, phase-coverage, etc.), the goodness-of-fit level has to be determined carefully to avoid the appearance of numerical artefacts in the final maps. This is particularly important in the case of resolution-limited maps (i.e. slowly rotating stars). However, there are only a few published procedures for establishing a robust stopping criterion, i.e. the point at which noise starts to affect the reconstructed image. Motivated by this, we propose a systematic method for estimating the optimal fit quality for a given set of ZDI magnetic field maps, using HD 1237 as a test-case. It is important to note here that since this procedure is defined a posteriori over the resulting maps themselves (2D images), it does not modify the regularisation functions imposed to ZDI. In this sense, its application to other stellar systems should be straightforward.

We begin with the definition of the entropy $S$, as an estimate of the information content in an image ${ }^{3}$. Following Sonka et al. (2007), let $P(k)$ be the probability that the difference between two adjacent pixels is equal to $k$. The image entropy can be estimated as

$S=-\sum_{k} P_{k} \log _{2}\left(P_{k}\right)$

where $\log _{2}$ is the base 2 logarithm. This implies that a larger or smaller amount of entropy in the image will depend on the contrast between adjacent pixels. An image that is perfectly constant will have an entropy of zero. For the methodology described below, we are not interested in the absolute values of the entropy, but rather its overall behaviour as a function of the reduced $\chi^{2}$.

For a given converged ZDI solution (i.e. a particular value of reduced $\left.\chi^{2}\right)$, we can calculate the total entropy content $\left(S_{\mathrm{T}}\right)$

\footnotetext{
3 A similar implementation of entropy is commonly used as a regularisation function in ZDI (see Piskunov \& Kochukhov 2002).
}

by applying the definition given by Eq. (9) to each one of the recovered maps:

$S_{\mathrm{T}}=S_{\mathrm{R}}+S_{\mathrm{M}}+S_{\mathrm{A}}$,

where $S_{\mathrm{R}}, S_{\mathrm{M}}$, and $S_{\mathrm{A}}$ represent the entropy contained in the radial, meridional, and azimuthal field components maps, respectively. Figure 6 shows in red the behaviour of $S_{\mathrm{T}}$ as a function of the reduced $\chi^{2}$ for the ZDI solution set of HD 1237.

As expected, by decreasing the reduced $\chi^{2}$, the information content in the resulting ZDI solution increases (field strength and structure). The overall behaviour of the total entropy content and information growth is consistent for both cases. In the case of the Gaussian profile (Fig. 6, left panel), the entropy growth remains fairly constant (close to zero) for high reduced $\chi^{2}$ values, reaching a maximum ${ }^{4}$ around $\chi^{2} \simeq 1.2$. However, by that point the concavity of the curve has changed (negative second derivative), suggesting a different regime for the information growth in the ZDI solutions. This is interpreted as a noise signature, reflected as artefacts in the final maps leading to an additional increment in the information growth. For this reason we adopt as optimal fit level the reduced $\chi^{2}$ value for which the rate of change in the information growth (second derivative) in the ZDI solution set is maximised. In this particular case, this occurs around $\chi^{2} \simeq 1.4$, as indicated by the fourth-order polynomial fit (segmented line) in the left panel of Fig. 6. A similar criterion plot is constructed for the Milne-Eddington line profile (Fig. 6, right panel), where a lower optimal reduced $\chi^{2} \simeq 1.1$ is achieved in this case $\mathrm{e}^{5}$.

\subsection{ZDI maps and synthetic Stokes $V$ profiles}

We reconstructed the ZDI surface magnetic field maps and the synthetic circularly polarised profiles based on the time series of LSD Stokes $V$ spectra. For this we used the cleaned-tweaked line mask (Sect. 5) and the stellar and line parameters derived in Sect. 6.1. The goodness-of-fit level in each case is selected under the criterion described in the last section.

Figures 7 and 8 show the results of the ZDI procedure for the first-epoch observations (2012 July) of HD 1237. Four vertical panels are presented where the first three correspond to the

4 The apparent negative sign in the first derivative is due to the reversed direction of the $x$-axis (reduced $\chi^{2}$ ).

5 The same criterion was applied to generate the magnetic field maps in the December dataset (Appendix A). An optimal reduced $\chi^{2} \simeq 0.6$ was obtained in this case, as expected for a dataset with fewer constraints. 

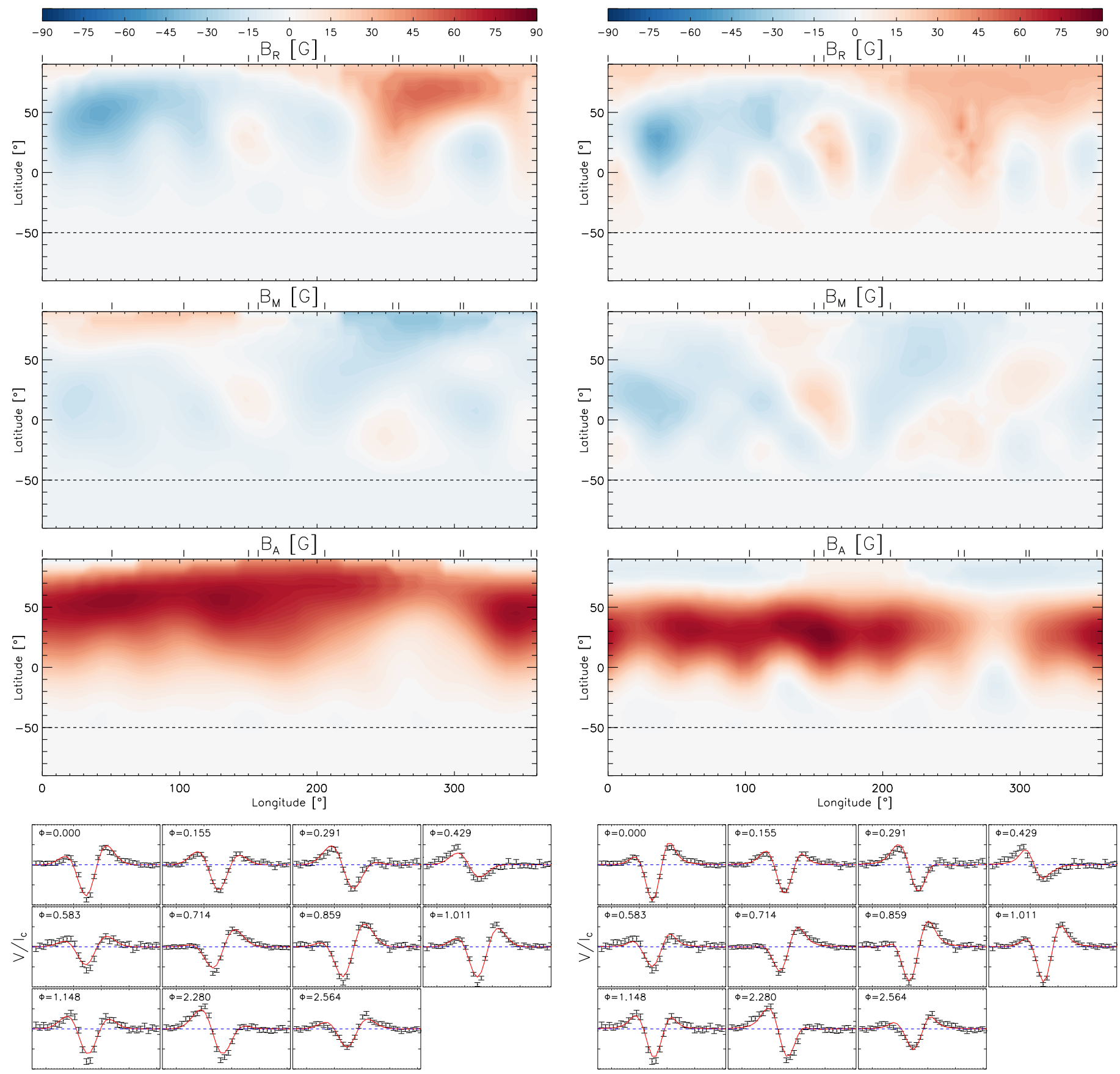

Fig. 7. Results of the ZDI analysis for the first-epoch observations of HD 1237 using the Gaussian line profile. The first three panels show the surface magnetic field components $B_{\mathrm{R}}, B_{\mathrm{M}}$, and $B_{\mathrm{A}}$. The colour scale indicates the polarity and the magnitude of the magnetic field component in $\mathrm{G}$, while the phase coverage is indicated by the black ticks in the upper $y$-axis. The segmented horizontal line indicates the surface visibility limit, imposed by the adopted inclination angle of the $\operatorname{star}\left(i=50^{\circ}\right)$. The last panel shows the comparison between synthetic (red) and observed (black) Stokes $V$ profiles obtained for this particular epoch in each observational phase $\Phi$, where the recovered maps fit the spectropolarimetric data to an optimal reduced $\chi^{2}=1.4$.

Mercator-projected magnetic field maps in Gauss (G), decomposed into the radial $\left(B_{\mathrm{R}}-\right.$ top), meridional $\left(B_{\mathrm{M}}-\right.$ middle), and azimuthal $\left(B_{\mathrm{A}}\right.$-bottom) components. The spatial resolution of the maps is $\sim 18^{\circ}$ in longitude at the stellar equator (at the poles, the map has a considerably poorer resolution than the equator). However, the phase coverage also has a significant impact on

Fig. 8. Results of the ZDI analysis for the first-epoch observations of HD 1237 using the Milne-Eddington line profile. See caption of Fig. 7 for more details. In this case, the maps fit the spectro-polarimetric data to an optimal reduced $\chi^{2}=1.1$.

the level of detail that can be recovered. In this case, the righthand side would have a slightly better resolution than the lefthand side of the image. The last panel shows the fitted synthetic Stokes $V$ profiles to the spectro-polarimetric observations in each rotational phase $(\Phi)$.

The recovered maps show a relatively complex field distribution across the surface. The field is dominated by the azimuthal component, displaying a strong $(\sim+90 \mathrm{G})$ large-scale ring-like structure around $45^{\circ}$ in latitude. In the radial component, two large and moderately strong $(\sim \pm 50 \mathrm{G})$ regions of opposite polarities are also located at higher latitudes, while weaker $(\sim \pm 25 \mathrm{G})$ small-scale features of mixed polarities appear close 
to the equator. These large magnetic features are somewhat preserved with reversed polarities in the meridional magnetic field maps. However, some cross-talk from the radial component may be present in the meridional map (see Donati \& Brown 1997).

Although the overall large-scale structure and field strength are consistent between both line profiles, several differences are clearly visible in the characteristics of the field components. First, the maps recovered using the Milne-Eddington line shape include additional small-scale features leading to a more complex field distribution in the surface. These are more prominent in the maps for the radial and meridional components. Second, the recovered magnetic field distribution, in the Milne-Eddington case, seems to be slightly shifted to lower latitudes. These differences can be understood from the fact that the Milne-Eddington line shape is a better representation of the derived LSD Stokes I profile. This is also true for the shape of the circularly polarised profile, leading to a more detailed (additional small-scale structures) and a field distribution shifted to lower latitudes (as a consequence of the sensitivity in the core of the profile). Both elements are translated into the lower optimal reduced $\chi^{2}$ value that can be reliably achieved in this case (see Sect. 6.2), and therefore an improved fit (lower panel in Fig. 8).

The reconstructed map for the second-epoch observations (2012 December) and the corresponding synthetic Stokes $V$ profiles are presented in Appendix A.

\section{Summary and discussion}

In this paper, we have presented a detailed study that covers two observational epochs of the activity and magnetic field structure of the Sun-like planet-hosting star HD 1237. The chromospheric activity level of HD 1237 estimated from the calibrated $S_{\mathrm{H}}=0.46 \pm 0.02$ and $\log \left(R_{H K}^{\prime}\right)=-4.38 \pm 0.05$ values is similar to other active Sun-like stars (e.g. $\xi$ boo A, Morgenthaler et al. 2012) and considerably higher than the solar case ( 3 times higher in terms of the average $S$ index, Lockwood et al. 2007). A much larger difference has been reported for the X-ray activity level, with a $\log \left(L_{\mathrm{X}}\right)=29.02 \pm 0.06$ for HD 1237 (Kashyap et al. 2008), which is two orders of magnitude higher than the value estimated for the Sun during solar maximum (Peres et al. 2000). The chromospheric activity level remained fairly constant over the near five-month period between the two sets of observations. Our estimate falls between the previous measurements of $\log \left(R_{H K}^{\prime}\right)=-4.27$ (Naef et al. 2001) and $\log \left(R_{H K}^{\prime}\right)=-4.44$ (Saffe et al. 2005). Given the large uncertainties in the activity indicators, it is difficult to address whether these variations have some correspondence to a magnetic cycle in the star.

To extract magnetic field signatures from spectropolarimetric data, we applied the LSD multi-line technique to the observations. Two different line masks for the LSD profiles were compared and tested. The standard procedure involves employing a mask "cleaned" of chromospheric and strong NLTE line profiles (e.g. Marsden et al. 2014); we compared the results from this procedure with improving the line list further by "tweaking" line strengths, so that they are tailored to the line depths in the observed spectrum. Both approaches lead to similar results in the obtained LSD profiles, and therefore in the physical quantities inferred (e.g. the longitudinal magnetic field, $B_{\ell}$ ). However, for the same number of spectral lines, the average $\mathrm{S} / \mathrm{N}$ of the LSD profiles recovered with the aid of the cleaned-tweaked line mask was $\sim 7 \%$ higher than in the cleaned case.

The longitudinal magnetic field $\left(B_{\ell}\right)$, estimated from the Stokes $I$ and $V$ LSD profiles, showed a clear rotational modulation with an amplitude of $\sim 10 \mathrm{G}$. This behaviour was preserved in both observed epochs. Placing these measurements in the context of other stars, similar variations have been observed in the long-term monitoring of the active Sun-like star $\xi$ Boo A ( 4-9 G, Age: 0.2 Gyr, Mamajek \& Hillenbrand 2008; Morgenthaler et al. 2012), the K-dwarf exoplanet host $\epsilon$ Eri ( 10-12 G, Age: $\sim 0.2-0.8$ Gyr, Janson et al. 2008; Jeffers et al. 2014b), and more recently for the young solar analogue HN Peg ( 14 G, Age: 0.2 Gyr, Eisenbeiss et al. 2013; Boro Saikia et al. 2015). The chromospheric and X-ray activity levels of these stars are also very similar to HD 1237 . The source $\xi$ Boo A has an average $S$-index of $S_{\mathrm{HK}} \simeq 0.45$ and $\log \left(L_{\mathrm{X}}\right) \simeq 28.91$ (Gray et al. 1996; Wood \& Linsky 2010), and $\epsilon$ Eri has strong magnetic activity with a mean $S_{\mathrm{HK}} \simeq 0.50$ and $\log \left(L_{\mathrm{X}}\right) \simeq 28.22$ (Jeffers et al. 2014b; Poppenhaeger et al. 2011). Similarly, previous reports for $\mathrm{HN}$ Peg show $S_{\mathrm{HK}} \simeq 0.35$ and $\log \left(L_{\mathrm{X}}\right) \simeq 29.19$ (Boro Saikia et al. 2015; Schmitt \& Liefke 2004). Despite the various similarities among these systems, the complete relation between the magnetic field and its influence over different layers of the stellar atmosphere (activity) have not yet been fully understood. Similar to these other systems, the chromospheric activity of HD 1237 does not show a clear correlation with $B_{\ell}$. This is interpreted as the result of probing different spatial and temporal scales in each of these measured quantities.

We recovered the optimal stellar parameters of HD 1237 using a reduced $\chi^{2}$ minimisation scheme, based on the tomographic inversion technique of ZDI. The analysis yields a rotation period, $P_{\text {rot }}=7.0 \pm 0.7$ days. Literature values of $P_{\text {rot }}$ are uncertain, ranging between $\sim 4.1-12.6$ days (see Watson et al. 2010 and references therein). The 7.0-day value found in this work is consistent with the ZDI analysis and both the chromospheric and coronal activity levels of the star. Similar procedures were applied to estimate the inclination angle and the differential rotation of HD 1237. However, the available observations did not provide enough constraints for a robust determination of these parameters. Therefore, we considered an inclination angle derived using the stellar properties of the star and solid body rotation (i.e. $i \sim 50^{\circ}$ ). No differential rotation profile was included in the reconstruction of the magnetic field maps.

For the surface magnetic field mapping procedure, two different synthetic line shapes (Gaussian/Milne-Eddington) were tested in order to investigate their impact on the ZDI maps for slowly rotating solar-type stars. We showed that both profiles recover robust magnetic field maps. However, the Milne-Eddington line profile yields a better spectro-polarimetric fit (lower optimal reduced $\chi^{2}$ ), leading to a more detailed structure recovered in the ZDI maps compared to the Gaussian case. In connection to this, a fit-stopping criterion based on the information content (entropy) of the ZDI maps solution set was introduced. This allows identification of the optimal reduced $\chi^{2}$ value, avoiding to some extent the appearance of numerical artefacts in the ZDI maps. The optimal fit level is given by the reduced $\chi^{2}$ value for which the rate of change of the entropy growth in the ZDI solution set is maximised.

The large-scale magnetic field of HD 1237 showed a complex distribution at the stellar surface. The strongest magnetic field features appear at middle latitudes $\left(\sim 45^{\circ}\right)$ in the azimuthal and radial components. The field is dominated by the azimuthal component, displaying latitudinal belts or ring-like structures across the stellar surface. This has been observed in other studies of active Sun-like stars (e.g. Folsom et al. 2014) and in numerical simulations of a rapidly rotating Sun (Brown et al. 2010). As previously suggested, the appearance of significant or even dominant toroidal fields in the surface of these types of stars is 
connected with the rotation period, with $\sim 12$ days as the rotation threshold (Petit et al. 2008).

In comparison with other cool stars, HD $1237\left(P_{\text {rot }}=\right.$ 7.0 days, this work, $M_{*}=1.0 \pm 0.1 M_{\odot}$, Ghezzi et al. 2010) could be located near $\xi$ Boo A (G8V, Age: $\sim 0.2$ Gyr) and GJ 182 (M0V, Age: $0.50_{-0.3}^{+1.0} \mathrm{Gyr}$, Liu et al. 2004) in the massperiod, large-scale magnetic field diagram (Fig. 3 in Donati \& Landstreet 2009) with a mostly toroidal field topology and a considerable contribution from the radial component. With the same spectral type as HD 1237, $\xi$ Boo A displays a slightly weaker field in its surface $(\sim \pm 60 \mathrm{G})$ with an alternating dominance between the radial and the azimuthal components in the observed long-trend evolution ( $\sim 4$ years, Morgenthaler et al. 2012). During the observed azimuthal-dominated epochs of this star, the field distribution is very similar to the one derived for HD 1237 in this work. A strong uni-directional azimuthal field appears at low latitudes with large mixed polarity regions in the radial field and a minor contribution from the meridional component. However, the magnetic regions of $\xi$ Boo A are much larger and less concentrated than the ones found for HD 1237. This could be related to the small difference in their rotation periods (6.43 days for $\xi$ Boo A and 7.0 days for HD 1237) and/or with the differential rotation that may be occurring in the surface. A similar situation appears in the case of GJ 182 in terms of the recovered magnetic field topology. However, the field strength is much larger in this case (up to $400 \mathrm{G}$, Donati et al. 2008b), which is mostly connected with the rapid rotation, large latitudinal shear, and the nearly fully convective nature of this star.

Regardless of the low $v \sin i$ of HD 1237, it is clear that a more complex field distribution is required to fit the observed spectra. Some weaker small-scale regions are recovered closer to the stellar equator. Despite their relative low strength compared to the total surface field, the contribution from these smallscale features to magnetically-related phenomena may be significantly higher. In combination with strong mixed-polarity regions missed by ZDI, these features can influence the quiescent coronal emission (Johnstone et al. 2010), the X-ray modulation (Arzoumanian et al. 2011), and the wind structure around very active stars (Lang et al. 2014) and the Sun (Garraffo et al. 2013).

The large-scale field distribution appears similar at both of the observed epochs, confirming the observed chromospheric activity and longitudinal magnetic field behaviour. Using the final ZDI maps and the derived synthetic Stokes $V$ profiles, we were able to reproduce the variations in $B_{\ell}$ consistently (for both observed epochs). As an example, the maximum value of $B_{\ell}$ in Fig. 4 coincides when the large positive polarity region of the radial field is located close to the limb (Day 3). Similarly, the minimum value obtained for $B_{\ell}$ in Fig. 4 results from the negative polarity region in the radial field map, this time located much closer to the disk centre. This corroborates the robustness of our ZDI magnetic maps for this system.

As mentioned in Sect. 2, HD 1237 has a planetary companion with a mean separation of $0.49 \mathrm{AU}$ and a projected mass of $M_{\mathrm{p}} \sin (i)=3.37 \pm 0.14 M_{4}$. This exoplanet is relatively far out in comparison with other similar systems where ZDI maps of the host star are available (see Fares et al. 2013 and references therein). However, the enhanced activity levels of HD 1237 and the relatively strong and complex surface magnetic field (in connection with its stellar wind) could affect the conditions experienced by the exoplanet through its orbit significantly. Previous parametric studies of this system have predicted a relatively high mass loss rate $\left(\sim 85 \dot{M}_{\odot}\right)$, which could even lead to magnetospheric radio emission from the exoplanet (Stevens 2005). This will be considered in a future study, incorporating the recovered ZDI magnetic field maps into a detailed 3D magnetohydrodynamics (MHD) code (BATS-R-US, Powell et al. 1999; Tóth et al. 2012), which was originally developed and validated for the solar wind and corona (e.g. Sokolov et al. 2013; van der Holst et al. 2014) and recently applied in the stellar context (e.g. Cohen \& Drake 2014; Cohen et al. 2014).

\section{Conclusions}

We reconstructed magnetic field maps of the young planethosting G-type star, HD 1237 using the technique of Zeeman Doppler imaging. As part of this detailed spectro-polarimetric study, we have performed the following.

- We find that assuming a Milne-Eddington approximation for the local line profile produces a better fit to the shape of the observed LSD profiles. This influences the number of magnetic structures that can be recovered in the magnetic field maps.

- We propose a robust method for defining the stopping criterion in ZDI techniques. This allows one to choose the optimum degree of fit, beyond which the model ceases to provide a good fit to the observed dataset owing to the introduction of artefacts into the resulting image. We successfully applied this to two different datasets with vastly different degrees of phase sampling.

- As part of our optimisation routines, we recovered a rotation period of 7.0 days. This is consistent with the measured chromospheric and coronal activity levels of the $\operatorname{star}\left(\log \left(R_{H K}^{\prime}\right)=\right.$ $-4.38 \pm 0.05$, this paper; $\log \left(L_{\mathrm{X}}\right)=29.02 \pm 0.06$, Kashyap et al. 2008).

- The magnetic field reconstructions for HD 1237 are dominated by a band of strong uni-directional azimuthal field at high latitudes, accompanied by a complex multi-polar radial field distribution. The largest magnetic regions show field strengths of $\sim 90$ and $50 \mathrm{G}$ for the azimuthal and the radial field components, respectively.

- We note that the field topology recovered for HD 1237 is fully commensurate with studies of $\xi$ Boo A and GJ 182, the two other stars that are closest to it in the mass, period diagram (Donati \& Landstreet 2009). Larger sample sizes are needed to confirm these trends. If confirmed, it will be possible to predict the global magnetic field topologies and therefore the extended environments of planet-hosting stars at various stages in their evolution based on these fundamental parameters.

We will address the influence of the different magnetic scales on the coronal structure and wind properties of HD 1237 in a follow-up paper, using the ZDI maps presented here as boundary conditions (see Cohen et al. 2010, 2011a). Possible starplanet interactions occurring in the system via transient (e.g. CME events, Cohen et al. 2011b) or quiescent phenomena (e.g. planetary radio emission, Stevens 2005; Vidotto et al. 2012) can also be considered in future work.

Acknowledgements. Based on observations made with ESO Telescopes at the La Silla Paranal Observatory under programme ID 089.D-0138.

\section{Appendix A: Second-epoch dataset (2012 Dec)}

We consider only the Milne-Eddington line profile for the 2012 Dec dataset. Figure A.1 contains the recovered ZDI maps for 


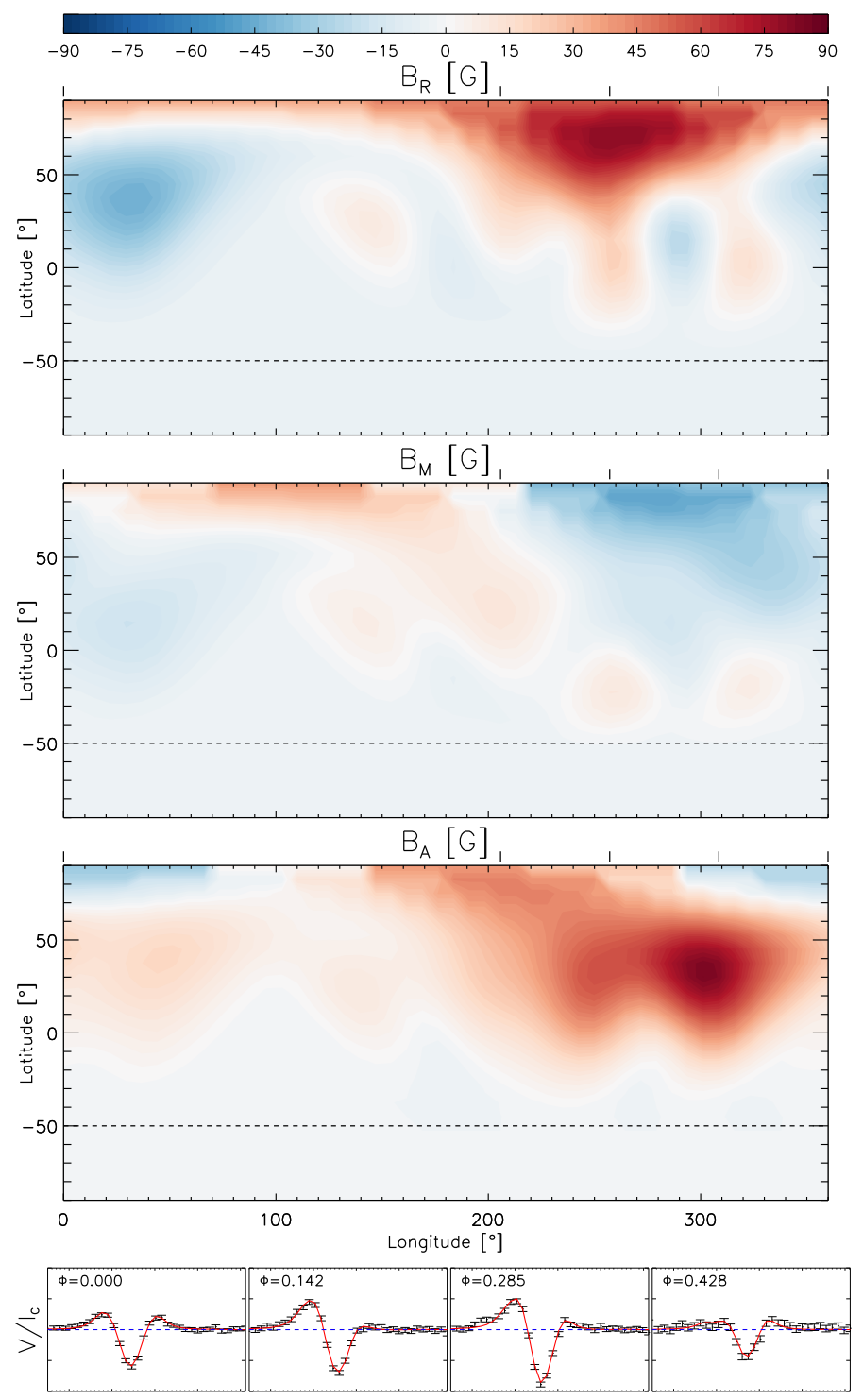

Fig. A.1. Results of the ZDI analysis for the second-epoch observations of HD 1237 using the Milne-Eddington line profile. See caption of Fig. 7 for more details. In this case the maps fit the spectro-polarimetric data to an optimal reduced $\chi^{2}=0.6$. In this case, $\Phi=0.0$ is assigned to the observations acquired at HJD 2456 265.0.

this epoch with the corresponding synthetic Stokes $V$ profiles. Despite having a lower phase coverage in this case, we were able to recover robust magnetic field maps, fitting the spectropolarimetric data up to an optimal reduced $\chi^{2}=0.6$ (see Sect. 6.2). This low value of reduced $\chi^{2}$ results as a consequence of the fewer constraints available for this dataset.

The field distribution clearly resembles the one obtained for the July dataset with a large contribution from the azimuthal and radial components to the total field. The main large-scale magnetic features are preserved between both observed epochs. This is consistent with the behaviour shown by the activity indicators (Sect. 4.2, Fig. 3) and the longitudinal magnetic field (Sect. 5.1, Fig. 4) in the entire dataset. Some of the smaller-scale structure is not recovered, and the ring of azimuthal field is not clear in this case. These changes are expected from the number of observations and phase coverage in this epoch (e.g. Donati \& Brown 1997). The slight shift in longitude is due to the initial phase selection in this case, where $\Phi=0.0$ is assigned to the observations acquired at HJD 2456265.0 (Table 2).

\section{References}

Anglada-Escudé, G., \& Butler, R. P. 2012, ApJS, 200, 15

Arzoumanian, D., Jardine, M., Donati, J.-F., Morin, J., \& Johnstone, C. 2011 , MNRAS, 410, 2472

Bagnulo, S., Landolfi, M., Landstreet, J. D., et al. 2009, PASP, 121, 993

Baliunas, S. L., Donahue, R. A., Soon, W. H., et al. 1995, ApJ, 438, 269

Barnes, J. R., Lister, T. A., Hilditch, R. W., \& Collier Cameron, A. 2004, MNRAS, 348, 1321

Bastien, F. A., Stassun, K. G., Pepper, J., et al. 2014, AJ, 147, 29

Boro Saikia, S., Jeffers, S. V., Petit, P., et al. 2015, A\&A, 573, A17

Brown, B. P., Browning, M. K., Brun, A. S., Miesch, M. S., \& Toomre, J. 2010, ApJ, 711, 424

Cohen, O., \& Drake, J. J. 2014, ApJ, 783, 55

Cohen, O., Drake, J. J., Kashyap, V. L., Hussain, G. A. J., \& Gombosi, T. I. 2010, ApJ, 721, 80

Cohen, O., Kashyap, V. L., Drake, J. J., et al. 2011a, ApJ, 733, 67

Cohen, O., Kashyap, V. L., Drake, J. J., Sokolov, I. V., \& Gombosi, T. I. 2011b, ApJ, 738, 166

Cohen, O., Drake, J. J., Glocer, A., et al. 2014, ApJ, 790, 57

Collier Cameron, A. 1995, MNRAS, 275, 534

Daou, A. G., Johns-Krull, C. M., \& Valenti, J. A. 2006, AJ, 131, 520

Donati, J.-F. 2003, in Solar Polarization, eds. J. Trujillo-Bueno, \& J. Sanchez Almeida, ASP Conf. Ser., 307, 41

Donati, J.-F., \& Brown, S. F. 1997, A\&A, 326, 1135

Donati, J.-F., \& Landstreet, J. D. 2009, ARA\&A, 47, 333

Donati, J.-F., Semel, M., Carter, B. D., Rees, D. E., \& Collier Cameron, A. 1997, MNRAS, 291, 658

Donati, J.-F., Jardine, M. M., Gregory, S. G., et al. 2008a, MNRAS, 386, 1234

Donati, J.-F., Morin, J., Petit, P., et al. 2008b, MNRAS, 390, 545

Donati, J.-F., Gregory, S. G., Alencar, S. H. P., et al. 2012, MNRAS, 425, 2948

Donati, J.-F., Hébrard, E., Hussain, G., et al. 2014, MNRAS, 444, 3220

Dumusque, X., Boisse, I., \& Santos, N. C. 2014, ApJ, 796, 132

Dunstone, N. J., Hussain, G. A. J., Collier Cameron, A., et al. 2008, MNRAS, 387,481

Eisenbeiss, T., Ammler-von Eiff, M., Roell, T., et al. 2013, A\&A, 556, A53

Ekenbäck, A., Holmström, M., Wurz, P., et al. 2010, ApJ, 709, 670

Fares, R. 2014, IAU Symp., 302, 180

Fares, R., Moutou, C., Donati, J.-F., et al. 2013, MNRAS, 435, 1451

Folsom, C. P., Petit, P., Bouvier, J., Donati, J.-F., \& Morin, J. 2014, IAU Symp., 302,110

Garraffo, C., Cohen, O., Drake, J. J., \& Downs, C. 2013, ApJ, 764, 32

Ghezzi, L., Cunha, K., Smith, V. V., et al. 2010, ApJ, 720, 1290

Gray, D. F., Baliunas, S. L., Lockwood, G. W., \& Skiff, B. A. 1996, ApJ, 465, 945

Hussain, G. A. J., Donati, J.-F., Collier Cameron, A., \& Barnes, J. R. 2000, MNRAS, 318, 961

Hussain, G. A. J., Collier Cameron, A., Jardine, M. M., et al. 2009, MNRAS, 398,189

Janson, M., Reffert, S., Brandner, W., et al. 2008, A\&A, 488, 771

Jeffers, S. V., Barnes, J. R., Jones, H. R. A., et al. 2014a, MNRAS, 438, 2717

Jeffers, S. V., Petit, P., Marsden, S. C., et al. 2014b, A\&A, 569, A79

Jensen, A. G., Redfield, S., Endl, M., et al. 2012, ApJ, 751, 86

Johnstone, C., Jardine, M., \& Mackay, D. H. 2010, MNRAS, 404, 101

Kashyap, V. L., Drake, J. J., \& Saar, S. H. 2008, ApJ, 687, 1339

Kochukhov, O., Makaganiuk, V., \& Piskunov, N. 2010, A\&A, 524, A5

Koen, C., Kilkenny, D., van Wyk, F., \& Marang, F. 2010, MNRAS, 403, 1949

Kotov, V. A., Scherrer, P. H., Howard, R. F., \& Haneychuk, V. I. 1998, ApJS, 116,103

Kupka, F. G., Ryabchikova, T. A., Piskunov, N. E., Stempels, H. C., \& Weiss, W. W. 2000, Balt. Astron., 9, 590

Lang, P., Jardine, M., Morin, J., et al. 2014, MNRAS, 439, 2122

Linsky, J. L., Yang, H., France, K., et al. 2010, ApJ, 717, 1291

Liu, M. C., Matthews, B. C., Williams, J. P., \& Kalas, P. G. 2004, ApJ, 608, 526 Lockwood, G. W., Skiff, B. A., Henry, G. W., et al. 2007, ApJS, 171, 260

Makaganiuk, V., Kochukhov, O., Piskunov, N., et al. 2011, A\&A, 525, A97

Mamajek, E. E., \& Hillenbrand, L. A. 2008, ApJ, 687, 1264

Markwardt, C. B. 2009, in Astronomical Data Analysis Software and Systems XVIII, eds. D. A. Bohlender, D. Durand, \& P. Dowler, ASP Conf. Ser., 411, 251

Marsden, S. C., Petit, P., Jeffers, S. V., et al. 2014, MNRAS, 444, 3517

Mayor, M., Pepe, F., Queloz, D., et al. 2003, The Messenger, 114, 20

Middelkoop, F. 1982, A\&A, 107, 31

Moré, J. 1978, in Lecture Notes in Mathematics, Numerical Analysis, ed. G. Watson (Berlin, Heidelberg: Springer), 630, 105

Morgenthaler, A., Petit, P., Saar, S., et al. 2012, A\&A, 540, A138

Morin, J., Donati, J.-F., Petit, P., et al. 2008, MNRAS, 390, 567

Naef, D., Mayor, M., Pepe, F., et al. 2001, A\&A, 375, 205 
Neiner, C., Grunhut, J. H., Petit, V., et al. 2012, MNRAS, 426, 2738

Noyes, R. W., Hartmann, L. W., Baliunas, S. L., Duncan, D. K., \& Vaughan, A. H. 1984, ApJ, 279, 763

Peres, G., Orlando, S., Reale, F., Rosner, R., \& Hudson, H. 2000, ApJ, 528, 537

Petit, P., Donati, J.-F., \& Collier Cameron, A. 2002, MNRAS, 334, 374

Petit, P., Dintrans, B., Solanki, S. K., et al. 2008, MNRAS, 388, 80

Piskunov, N., \& Kochukhov, O. 2002, A\&A, 381, 736

Piskunov, N. E., \& Valenti, J. A. 2002, A\&A, 385, 1095

Piskunov, N., Snik, F., Dolgopolov, A., et al. 2011, The Messenger, 143, 7

Poppenhaeger, K., Robrade, J., \& Schmitt, J. H. M. M. 2011, A\&A, 529, C1

Powell, K. G., Roe, P. L., Linde, T. J., Gombosi, T. I., \& De Zeeuw, D. L. 1999 ,

J. Comput. Phys., 154, 284

Saffe, C., Gómez, M., \& Chavero, C. 2005, A\&A, 443, 609

Santos, N. C., Mayor, M., Naef, D., et al. 2000, A\&A, 361, 265

Sanz-Forcada, J., Micela, G., Ribas, I., et al. 2011, A\&A, 532, A6

Schmitt, J. H. M. M., \& Liefke, C. 2004, A\&A, 417, 651

Schröder, C., Reiners, A., \& Schmitt, J. H. M. M. 2009, A\&A, 493, 1099

Semel, M. 1989, A\&A, 225, 456

Shibata, K., \& Magara, T. 2011, Liv. Rev. Sol. Phys., 8, 6
Sing, D. K. 2010, A\&A, 510, A21

Sokolov, I. V., van der Holst, B., Oran, R., et al. 2013, ApJ, 764, 23

Sonka, M., Hlavac, V., \& Boyle, R. 2007, Image Processing, Analysis, and

Machine Vision (Thomson-Engineering)

Stevens, I. R. 2005, MNRAS, 356, 1053

Torres, C. A. O., Quast, G. R., da Silva, L., et al. 2006, A\&A, 460, 695

Tóth, G., van der Holst, B., Sokolov, I. V., et al. 2012, J. Comput. Phys., 231, 870

van der Holst, B., Sokolov, I. V., Meng, X., et al. 2014, ApJ, 782, 81

Vidal-Madjar, A., Lecavelier des Etangs, A., Désert, J.-M., et al. 2003, Nature, 422,143

Vidotto, A. A., Fares, R., Jardine, M., et al. 2012, MNRAS, 423, 3285

Vidotto, A. A., Jardine, M., Morin, J., et al. 2013, A\&A, 557, A67

Vogt, S. S., Penrod, G. D., \& Hatzes, A. P. 1987, ApJ, 321, 496

Watson, C. A., Littlefair, S. P., Collier Cameron, A., Dhillon, V. S., \& Simpson, E. K. 2010, MNRAS, 408, 1606

Wood, B. E. 2004, Liv. Rev. Sol. Phys., 1, 2

Wood, B. E., \& Linsky, J. L. 2010, ApJ, 717, 1279

Zechmeister, M., \& Kürster, M. 2009, A\&A, 496, 577 